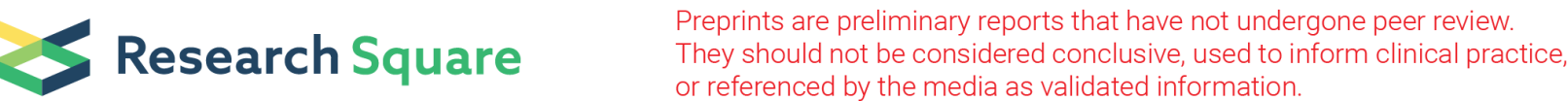

\section{Neoadjuvant Weekly Paclitaxel and Carboplatin with Trastuzumab and Pertuzumab in HER2-positive Breast Cancer: A Brown University Oncology Research Group (BrUOG) Study}

Mary Lorraine Lopresti

Miriam Hospital

Jessica J Bian

Maine Medical Center

Bachir Joseph Sakr

Women and Infants Hospital of Rhode Island

Rochelle S Strenger

Miriam Hospital

Robert Duffy Legare

Yale New Haven Health Smilow Cancer Hospital

Mary Anne Fenton

Rhode Island Hospital

Sabrina M Witherby

Miriam Hospital

Donald S Dizon

Rhode Island Hospital

Sonali V Pandya

Beth Israel Deaconess Medical Center

Ashley R Stuckey

Women and Infants Hospital of Rhode Island

David A Edmondson

Women and Infants Hospital of Rhode Island

Jennifer S Gass

Women and Infants Hospital of Rhode Island

Christine M Emmick

Miriam Hospital

Theresa A Graves

Rhode Island Hospital

Marlene Cutitar

Miriam Hospital 


\section{Adam J Olszewski}

Rhode Island Hospital

William M Sikov (D wsikov@wihri.org)

Women and Infants Hospital of Rhode Island https://orcid.org/0000-0001-5451-6449

\section{Research Article}

Keywords: Breast cancer, early stage, HER2-positive breast cancer, neoadjuvant chemotherapy, carboplatin

Posted Date: March 24th, 2021

DOl: https://doi.org/10.21203/rs.3.rs-346637/v1

License: (c) (i) This work is licensed under a Creative Commons Attribution 4.0 International License.

Read Full License 


\section{Abstract}

\section{Purpose:}

In HER2-positive breast cancer (HER2+BC), neoadjuvant chemotherapy (NACT) with dual HER2-targeted therapy achieves high pathologic complete response (pCR) rates. Anthracycline-free NACT regimens avoid toxicities associated with anthracyclines, but every 3-week TCHP also has substantial side effects. We hypothesized that a weekly regimen might have equivalent efficacy with less toxicity; we also investigated whether poorly responding patients would benefit from switching to AC.

\section{Methods:}

Patients with clinical stage II-III HER2+BC received weekly paclitaxel $80 \mathrm{mg} / \mathrm{m}^{2}$ and carboplatin AUC2 with every 3-week trastuzumab and pertuzumab (wPCbTP), with the option of splitting the pertuzumab loading dose. After 12 weeks, responding patients continued wPCbTP for another 6 weeks, while nonresponders switched to AC. Dose modifications and post-op therapy were at investigator discretion.

\section{Results:}

In 30 evaluable patients, the pCR rate was 77\% (95\% Cl 58-90\%); 12/14 (86\%) in ER-negative and 11/16 (69\%) in ER-positive. Only two patients transitioned to AC for non-response, of which one achieved pCR. There were no episodes of febrile neutropenia or grade $\geq 3$ peripheral neuropathy, though several patients who continued wPCbTP stopped before week 18. Split-dose pertuzumab was associated with less grade $\geq 2$ diarrhea (40\%) than the standard loading dose (60\%).

\section{Conclusions:}

PCR rates with our regimen were as high as reported with TCHP with fewer grade $\geq 3$ toxicities, though diarrhea remains a concern. Too few patients had a suboptimal response to adequately test switching to AC. The wPCbTP regimen should be considered an alternative to TCHP as neoadjuvant therapy for HER2+BC.

\section{ClinicalTrials.gov identifier: NCT02789657}

\section{Background And Rationale}

Neoadjuvant chemotherapy with dual HER2-targeted therapy achieves high pathologic complete response ( $\mathrm{pCR}$, ypT0/isN0) rates in patients with HER2-positive breast cancer (HER2+BC), and patients who achieve PCR have excellent long-term outcomes [1,2]. However, the optimal chemotherapy regimen to partner with the HER2-targeted agents, in terms of both efficacy and toxicity, is unclear. In the adjuvant setting, administration of an anthracycline with subsequent taxane and trastuzumab has been associated with increased risks of cardiac toxicity and leukemogenesis, while an anthracycline-free 
regimen consisting of docetaxel, carboplatin and trastuzumab $(\mathrm{TCH})$ achieved similar long-term breast cancer-free outcomes without these additional toxicities [3].

The TRYPHAENA randomized phase II neoadjuvant trial demonstrated that administration of an anthracycline-free regimen ( 6 cycles of every-3-week docetaxel, carboplatin, trastuzumab and pertuzumab, often referred to as TCHP) resulted in a PCR rate at least equivalent to that seen with an anthracycline-containing regimen (fluorouracil, epirubicin and cyclophosphamide followed by docetaxel with trastuzumab and pertuzumab, FEC-THP) (66\% vs. $57 \%$, respectively) [4]. Three-year disease-free survival (DFS) rates were also similar, at $90 \%$ for TCHP vs. $88 \%$ for FEC-THP [5]. However, these results do not rule out the possibility that there exists a subset of non-metastatic HER2+ patients who would benefit from receiving an anthracycline. In addition, standard TCHP is associated with sufficient myelosuppression to require prophylactic myeloid growth factor support and not infrequent grade 3 diarrhea [4]. Regimens substituting weekly paclitaxel (wP) for docetaxel in combination with trastuzumab $(T)$ and pertuzumab $(P)$ have demonstrated equivalent efficacy with a more favorable toxicity profile in HER2+ metastatic breast cancer [6]. A prior study from our group demonstrated that the combination of WP and every 4-week carboplatin (Cb), dosed at area-under-the-curve (AUC) 6 with T (and without subsequent administration of an anthracycline) achieved a pCR rate of 75\% [7]. Based on our experience in patients with both HER2+BC and triple-negative breast cancer, we believed that substituting lower dose (AUC 2) weekly $\mathrm{Cb}$ for higher dose every 3- or 4-week $\mathrm{Cb}$ could reduce toxicity and improve adherence to the planned treatment schedule.

The primary objectives of this pilot study were to assess the efficacy and tolerability of weekly paclitaxel and carboplatin with trastuzumab and pertuzumab (wPCbTP) as neoadjuvant therapy for stage II-III HER2+ breast cancer, and to determine whether patients who have evidence of a suboptimal clinical response after 12 weeks benefit from switching to an anthracycline-containing regimen, as assessed by achievement of pCR.

\section{Patients And Methods}

Eligible patients had histologically confirmed adenocarcinoma of the breast that was HER2+ as defined by either a score of $3+$ on immunohistochemical staining or amplification of the HER2 gene by FISH or $\mathrm{CISH}$ analysis (defined as a HER2/CEP17 ratio $\geq 2.0$ or average number of HER2 copies per nucleus of $>6$ ). Patients were clinical stage IIA-IIIC and were stratified as operable (stage II-IIIA) or inoperable (IIIB-C). Patients with stage IV (metastatic) disease, occult primary breast cancers (TX) or breast tumors $<1 \mathrm{~cm}$ in greatest dimension were not eligible even with histologically confirmed nodal involvement. Patients with multifocal disease in the same breast were eligible if all sampled lesions were HER2+. Patients with DCIS or clinical $\mathrm{T} 1 \mathrm{a} / \mathrm{bN} 0$ disease in the contralateral breast were eligible regardless of receptor status, but $\mathrm{pCR}$ was determined solely on histologic response in the HER2+ target lesion. Patients had to be female and $\geq 18$ years of age, with a Zubrod PS of 0-1 and adequate hematologic, renal, hepatic and cardiac function. Patients were excluded if they had received prior treatment for this cancer, had poorly controlled cardiovascular disease or had experienced a major cardiovascular event within the past year, were 
pregnant or lactating, or had grade $>1$ baseline peripheral neuropathy. The study was approved by the institutional review boards at all participating hospitals and all patients provided a signed informed consent.

\section{Treatment Plan}

Patients received paclitaxel $80 \mathrm{mg} / \mathrm{m}^{2}$ and carboplatin AUC 2 weekly for 12 weeks, with trastuzumab 8 $\mathrm{mg} / \mathrm{kg}$ week 1 then $6 \mathrm{mg} / \mathrm{kg}$ every 3 weeks and pertuzumab $840 \mathrm{mg}$ week 1 then $420 \mathrm{mg}$ every 3 weeks (wPCbTP), though treating physicians had the option of splitting the loading dose of pertuzumab into two doses of $420 \mathrm{mg}$ administered weeks 1 and 2, to determine if this approach might reduce toxicities, especially diarrhea, during the first 6 weeks of treatment (see Schema, Figure 1). After 12 weeks of chemotherapy, which could extend beyond 12 weeks of treatment if the patient required one or more treatment delays (see below), patients underwent imaging - usually an ultrasound of the involved breast, and ipsilateral axilla if node-positive at baseline. Patients who met criteria for clinical CR or PR, with a decrease in the product of the two largest diameters of the breast mass of $>50 \%$, continued the WPCbTP regimen for another 6 weeks. Patients with a suboptimal response to treatment, and those in whom the treating physician believed that continuation of the WPCbTP regimen was contraindicated due to toxicity, could be taken to surgery at that point or switched to doxorubicin $60 \mathrm{mg} / \mathrm{m}^{2}$ and cyclophosphamide 600 $\mathrm{mg} / \mathrm{m}^{2}$, with prophylactic pegfilgrastim, every 2 weeks for 4 cycles (ddAC). Trastuzumab and pertuzumab were held during ddAC.

Patients were assessed for eligibility for breast-conserving surgery and planned management of the axilla prior to starting treatment and again prior to surgery. Patients underwent surgery 3-6 weeks after the last dose of neoadjuvant therapy. Surgical management was at the discretion of the treating physicians, but excision of the primary breast tumor and evaluation of the ipsilateral axillary lymph nodes were required. Pathologic response to treatment was assessed by the institutional pathologists, including assessments necessary to calculate residual cancer burden (RCB), as described by Symmans and colleagues [8], in patients with residual disease. Post-op treatment, including radiation, adjuvant chemotherapy, adjuvant endocrine therapy in hormone receptor-positive $(\mathrm{HR}+)$ patients, resumption of trastuzumab +/pertuzumab or administration of other treatment, was at the discretion of the treating physicians. Study monitoring ended after a follow-up visit 3 months after surgery to record post-op treatments and assess for any late toxicities.

\section{Dose Modifications:}

Treating oncologists were strongly encouraged to complete 12 weeks of wPCb chemotherapy, with treatment delays or other modifications, including addition of filgrastim injections for severe or persistent neutropenia, permitted as deemed necessary. While essentially at physician discretion, treatment modifications were recommended for neutropenia $\left(\right.$ ANC $<800 / \mathrm{mm}^{3}$ ), thrombocytopenia (platelets $<50,000 / \mathrm{mm}^{3}$ ) or persistent grade $\geq 2$ non-hematologic toxicities, such as diarrhea or neuropathy, on the day of treatment, or for serious or life-threatening toxicity, such as febrile neutropenia, bleeding requiring 
transfusion or grade $\geq 3$ diarrhea, during the prior cycle. Administration of trastuzumab and pertuzumab continued every 3 weeks if chemotherapy was delayed, though treatment with trastuzumab or pertuzumab could also be delayed, held or even discontinued for severe or persistent toxicities ascribed to these agents, such as diarrhea attributed to pertuzumab. Treatment delays or dose modifications during ddAC were also at the discretion of the treating physician.

The primary endpoint of the study was to estimate the PCR rate achieved with the wPCbTP treatment regimen, including results in patients who were switched to ddAC, which included calculating the ClopperPearson binomial confidence interval for that rate.

\section{Results}

Thirty-two patients were enrolled on the study at the Breast Health Center of Women and Infants Hospital of Rhode Island and the Lifespan Comprehensive Cancer Centers at Rhode Island and Miriam Hospitals between November 2016 and June 2018, of whom 30 were evaluable for response and toxicity (two patients refused continuation of study treatment after just a few doses and thus were not considered evaluable) (see CONSORT diagram, Figure 2). Median age was 53 (range, 29-81), the study population was almost evenly divided between patients with ER-positive (16) and ER-negative (14) tumors. At baseline 22 patients had operable disease (clinical stage IIA-IIIA), 8 had inoperable disease (clinical stage IIIB-C) and 19 were clinically node-positive. In 20 patients the treating physicians chose to split the loading dose of pertuzumab between weeks 1 and 2 .

\section{Treatment Delivery and Toxicity}

Treatment delivery is summarized in Table 1. All 30 patients completed the planned first 12 weeks of wPCbTP. At that point, 2 patients discontinued treatment and went to surgery, 2 patients were switched to ddAC for a suboptimal response and one was switched for toxicity. Of 25 patients who continued WPCbTP, treatment was discontinued prior to week 18 in 9 due to toxicity, patient preference or physician decision. Among patients who continued treatment beyond week 12, the median number of doses delivered was 18 for paclitaxel and 17 for carboplatin. The paclitaxel dose was reduced in 8 patients for peripheral neuropathy and in one each for neutropenia and anemia. Carboplatin was discontinued early in 2 patients for hypersensitivity reactions (both after week 8), and in one each for diarrhea and neutropenia. One or more doses of pertuzumab were held in 8 patients for diarrhea, and this agent was discontinued in 6 for the same reason.

After surgery most $(28 / 30)$ patients received breast or chest wall and regional nodal irradiation. All patients with $\mathrm{HR}+$ cancers started adjuvant endocrine therapy. All patients resumed trastuzumab in the adjuvant setting, except for 2 patients who instead received adjuvant ado-trastuzumab emtansine (TDM1) once results of the KATHERINE trial [9] became available. No patient received adjuvant pertuzumab. 
The most common reported toxicities were neutropenia, anemia and diarrhea (Table 2). The most common grade $\geq 3$ toxicities were neutropenia $(n=17)$, anemia (4) and diarrhea (5). There were no instances of febrile neutropenia or grade $\geq 3$ peripheral neuropathy, though 5 patients developed grade 2 neuropathy. Adverse events requiring hospitalization included diarrhea (2, in one instance with documented clostridium difficile infection) and one instance each of atrial fibrillation, recurrent urinary tract infections associated with kidney stones, breast and port site infections while the patients were not neutropenic. The incidence of grade $\geq 2$ diarrhea was lower in patients who received the split loading dose of pertuzumab $(8 / 20,40 \%)$ than those who received the full dose week $1(6 / 10,60 \%)$.

\section{Efficacy}

Pathologic responses at surgery are tabulated in Table 3. Overall, 23 of 30 evaluable patients $(77 \%, 95 \%$ $\mathrm{Cl} 58-90 \%)$ achieved a pCR. The pCR rate was higher in estrogen receptor (ER)-negative patients (12/14, $86 \%)$ than in ER-positive patients $(11 / 16,69 \%)$, though with overlapping confidence intervals in this small study. Patients with operable disease at baseline had a higher pCR rate $(19 / 22,86 \%)$ than those with inoperable disease $(4 / 8,50 \%)$. Among the 3 patients switched to AC, 2 (one switched for toxicity and one for inadequate response on re-staging) achieved pCR. Among patients with residual disease at surgery, 3 met criteria for RCB class I, thus the pCR + RCBI rate was 26/30 (87\%), and the other 3 were RCBII.

At restaging following week 12, 11 patients were considered to have achieved a clinical CR, of whom 9 $(82 \%)$ had a pCR at surgery, 14 had a clinical PR, of whom 12 (86\%) had a pCR at surgery, and 5 had a clinical minor response or stable disease, of whom $2(40 \%)$ had a pCR at surgery.

Of 16 patients who were not considered breast conservation candidates at baseline, 3 converted to breast-conserving surgery (BCS)-eligible with neoadjuvant therapy. Overall, 15 patients had breastconserving surgery and 15 had a mastectomy (2 patients considered BCS-eligible opted for mastectomy, one in the setting of a deleterious mutation in BRCA2). Of 18 patients who were judged prior to treatment to require an axillary lymph node dissection, half (9) converted to clinically node-negative with neoadjuvant therapy and underwent sentinel lymph node biopsy.

\section{Discussion}

In this single arm pilot study, the combination of weekly paclitaxel and carboplatin with trastuzumab and pertuzumab yielded an impressive $77 \% \mathrm{pCR}$ rate. While our study is too small to draw any definitive conclusions, our results are at least comparable to those achieved in larger trials on which patients received a carboplatin-containing chemotherapy regimen and dual HER2-targeted therapy (Table 4). While we are not collecting long-term outcomes such as disease recurrence or survival, based on results from prior studies we would expect our patient population, particularly those who achieved pCR, to have a very good prognosis.

Our regimen and results are most comparable to those reported from the phase 3 TRAIN-2 trial conducted by the Dutch Breast Cancer Research Group (BOOG), with similar pCR rates, overall and in the ER- and 
ER+ subsets. That study randomized 438 patients to receive either 3 cycles of fluorouracil, epirubicin and cyclophosphamide with trastuzumab and pertuzumab (FEC-TP) followed by 6 cycles of paclitaxel days 1 and 8 with carboplatin AUC 6, trastuzumab and pertuzumab (wPq3CbTP) on day 1 every 3 weeks for 6 cycles (a total of 12 doses of weekly paclitaxel and 6 of carboplatin) or the anthracycline-free regimen of wPq3CbTP for 9 cycles (18 doses of weekly paclitaxel and 9 of carboplatin). In 2018 they reported equivalent pCR rates across the two arms (67\% and 68\%), and in 2020 they reported equivalent 3-year event-free survival (EFS) (92.7\% vs. 93.5\%) and overall survival (OS) (97.7\% vs. 98.2\%) with FEC-TP $\rightarrow$ wPq3CbTP and wPq3CbTP, respectively $[10,11]$. In neither analysis was a patient subgroup identified who had a superior PCR rate or improved long-term outcomes with inclusion of the anthracycline-containing regimen. Most acute toxicities between the two arms were comparable, but there was a significantly higher rate of febrile neutropenia in the anthracycline-containing arm ( $10 \% \mathrm{vs.} 1 \%, \mathrm{p}<.05)$ and nonsignificantly higher rates of grade $\geq 3$ diarrhea ( $17 \%$ vs. $12 \%$ ) and peripheral neuropathy $(7 \%$ vs. $5 \%$ ) in patients assigned to 9 cycles of WPq3CbTP. In addition, rates of cardiotoxicity differed significantly, with a left ventricular ejection fraction drop of at least $10 \%$ or to $<50 \%$ in $36 \%$ and meeting both criteria in $8 \%$ with the anthracycline-containing regimen compared to $22 \%$ and $3 \%$, respectively, on the anthracyclinefree arm. Moreover, two patients (1\%) on the anthracycline-containing arm have developed acute leukemia during follow-up compared to none on the anthracycline-free arm. While these results do not rule out the possibility that there is a subset of patients with stage II-III HER2+ breast cancer who would benefit from inclusion of an anthracycline in their neoadjuvant (or adjuvant) chemotherapy regimen, unless or until such patients can be identified many breast oncology experts have concluded that an anthracycline-free regimen should be the treatment of choice in these patients. While one of the objectives of our pilot study was to try to identify such patients, the observation that only two patients transitioned to ddAC after 12 weeks due to lack of response to WPCbTP supports the hypothesis that the group of patients who would benefit from inclusion of an anthracycline must be small, if it exists. Moreover, of these two patients, the one who was found to have achieved a PCR was switched to ddAC because of a persistent breast mass which did not decrease in size during ddAC and was demonstrated to be a large area of fibrosis without viable residual invasive disease at surgery, raising the possibility that she achieved pCR with the wPCbTP regimen and may not have benefited from the ddAC.

There are two major differences between the anthracycline-free regimen used in TRAIN-2 and the one in our study. First, they administered carboplatin at AUC 6 every 3 weeks for a total of 9 doses, while we administered carboplatin AUC 2 weekly for 18 doses. Since patients are coming in for weekly paclitaxel treatments anyway, there is minimal added inconvenience from the weekly carboplatin dosing. While it is difficult to compare toxicities across studies, there did not appear to be a difference in the incidence of grade $\geq 3$ neutropenia (TRAIN-2 - 54\%, BrUOG $308-57 \%$ ) or diarrhea (17\% in both studies), but we observed a much lower incidence of grade $\geq 3$ thrombocytopenia ( $3 \%$ vs. $19 \%$ ) and no instances of grade $\geq 3$ peripheral neuropathy (vs. 7\% on the wPq3CbTP arm of TRAIN-2). Perhaps related to the increased number of carboplatin doses administered, two (7\%) of our patients developed hypersensitivity reactions to carboplatin, which is usually seen only after multiple doses of this agent, requiring its discontinuation; the TRAIN-2 investigators do not report whether they observed this toxicity. Second, their treatment 
schedule had built-in breaks every 3 weeks (since paclitaxel was administered only days 1 and 8 every 3 weeks) while on our study treatment was given every week, with treatment delays permitted as needed for hematologic or non-hematologic toxicities. As a result, while our patients usually finished their neoadjuvant therapy in $<20$ weeks, the TRAIN-2 regimen takes a minimum of 27 weeks to complete, though which approach might be preferable to a patient is not known.

The most common serious toxicity observed with the addition of pertuzumab to chemotherapy + trastuzumab is diarrhea, which most often occurs during the first 6 weeks of treatment. The etiology of this toxicity is unknown, but the assumption is that it reflects interference of normal physiologic activity of the HER2:HER3 heterodimer, and signaling initiated by the HER2 tyrosine kinase, in the normal gut, likely exacerbated by chemotherapeutic agents, perhaps especially carboplatin. The rationale for the standard double 'loading' dose of pertuzumab administered week 1 is to rapidly achieve a steady state serum concentration to inhibit HER2:HER3 heterodimer formation, but we hypothesized that the higher serum levels associated with this larger dose might contribute to the frequency and severity of this toxicity and that the desired steady state concentration would likely be reached almost as quickly if the loading dose were split between weeks 1 and 2. This approach appeared to reduce the incidence of grade $\geq 2$ diarrhea (40\%) compared to patients who received the single loading dose (60\%), but a much larger randomized trial comparing these dosing schedules would be needed to determine if this is a worthwhile substitution.

Our study supports the use of an anthracycline-free neoadjuvant chemotherapy regimen with dual HER2targeted therapy in stage II-III HER2+ breast cancer, demonstrating high pCR rates and a high rate of conversion of node-positive patients to clinically node-negative (50\%), making them candidates for more limited axillary lymph node dissection. Given the impressive antitumor activity of combining cytotoxic chemotherapy with effective HER2 blockade, the CompassHER-pCR trial (EA1181, NCT04266249) is assessing the $\mathrm{PCR}$ rate of single agent WP combined with $\mathrm{T}$ and $\mathrm{P}$ for 12 weeks in these patients. While the overall pCR rate achieved with this regimen, which omits carboplatin and reduces the duration of treatment to 12 weeks, is certain to be lower than that achieved with a regimen such as ours, the goal of this trial is to try to identify a subset of patients who will achieve pCR with less intensive treatment. The study assumes that patients who fail to achieve a pCR can be effectively salvaged with adjuvant chemotherapy, adjuvant endocrine therapy (in HR+ patients) and/or adjuvant T-DM1, though a larger proportion of patients is expected to receive this agent, extending the duration of cytotoxic therapy and increasing costs.

In conclusion, standard neoadjuvant treatment for clinical stage II-III HER2+BC should be an anthracycline-free, carboplatin-containing NACT regimen with dual HER2-targeted therapy with trastuzumab and pertuzumab. Given the manageable toxicities and impressive pCR results achieved with the WPCbTP regimen, this has become our preferred neoadjuvant regimen in these patients.

\section{Abbreviations}


BrUOG: Brown University Oncology Research Group

HER2+BC: Human epidermal growth factor receptor 2 - positive breast cancer

NACT: Neoadjuvant chemotherapy

pCR: Pathologic complete response

TCHP: Every 3-week docetaxel, carboplatin, trastuzumab and pertuzumab

wPCbTP: Weekly paclitaxel and carboplatin with every 3-week trastuzumab and pertuzumab

AC: Doxorubicin and cyclophosphamide; ddAC: Every 2-week AC with pegfilgrastim

ER-positive/ER-negative: Estrogen receptor-positive/-negative

TCH: Every 3-week docetaxel, carboplatin and trastuzumab

FEC-THP: Fluorouracil, epirubicin and cyclophosphamide followed by docetaxel, trastuzumab and pertuzumab

wP: Weekly paclitaxel; Cb: Carboplatin; T: Trastuzumab; P: Pertuzumab

AUC: Area-under-the-curve

CEP17: Centromeric region of chromosome 17

$\mathrm{FISH} / \mathrm{CISH}$ : Fluorescent/chromogenic in situ hybridization

PS: Performance status

DCIS: Ductal carcinoma in situ

ANC: Absolute neutrophil count

T-DM1: Ado-trastuzumab emtansine

Cl: Confidence interval

RCB: Residual cancer burden

CR: Complete response; PR: Partial response

BRCA2: Breast cancer susceptibility gene 2

HER3: Human epidermal growth factor receptor 3

BO0G: Dutch Breast Cancer Research Group 


\section{Declarations}

\section{Funding:}

This research was supported by LifeCycle and the Lura Cook Hull Trust

\section{Conflicts of interest/Competing interests:}

The authors have no disclosures or conflicts of interest related to this manuscript to declare.

\section{Availability of data:}

Access to the supporting data for this study will be made available through the Brown University Oncology Research Group (BrUOG) upon request.

\section{Author contributions:}

All authors contributed to the study conception and design and/or its conduct and analysis. Data analysis was performed by William Sikov, Mary Lopresti and Jessica Bian, with statistical assistance from Adam Olszewski. The first draft of the manuscript was written by William Sikov and all authors commented on previous versions of the manuscript. All authors read and approved the final manuscript.

\section{Ethics approval/Consent to participate:}

The study was approved by the institutional review boards at all participating hospitals and all patients provided signed informed consent.

\section{Disclosures:}

The authors note no disclosures or conflicts of interest pertinent to this publication

\section{Acknowledgements and Funding Sources:}

We would like to express our appreciation for the efforts of the staff at the Brown University Oncology Group (BrUOG) administrative office, the research staff at the participating centers, and to all the patients who agreed to participate in the study. This study was supported by grants from LifeCycle and the Lura Cook Hull Trust.

\section{References}

1. Cortazar P, Zhang L, Untch M, et al (2014). Pathological complete response and long-term clinical benefit in breast cancer: the CTNeoBC pooled analysis. Lancet 384: 164-172.

2. Spring LM, Fell G, Arfe A, et al (2020). Pathological complete response after neoadjuvant chemotherapy and impact on breast cancer recurrence and survival: a comprehensive meta-analysis. Clin Cancer Res 26: 2838-2848. 
3. Slamon D, Eiermann W, Robert N, et al (2011). Adjuvant trastuzumab in HER2-positive breast cancer. N Engl J Med 365:1273-1283.

4. Schneeweiss A, Chia S, Hickish T, et al (2013). Pertuzumab plus trastuzumab in combination with standard neoadjuvant anthracycline-containing and anthracycline-free chemotherapy regimens in patients with HER2-positive early breast cancer: a randomized phase II cardiac safety study (TRYPHAENA). Ann Oncol 24:2278-2284.

5. Schneeweiss A, Chia S, Hickish T, et al (2018). Long-term efficacy analysis of the randomised, phase II TRYPHAENA cardiac safety study: Evaluating pertuzumab and trastuzumab plus standard neoadjuvant anthracycline-containing and anthracycline-free chemotherapy regimens in patients with HER2-positive early breast cancer. Eur J Cancer 89:27-35.

6. Dang C, lyengar N, Datko F et al (2015). Phase II study of paclitaxel given once per week along with trastuzumab and pertuzumab in patients with human epidermal growth factor receptor 2-positive metastatic breast cancer. J Clin Oncol33:442-447.

7. Sikov WM, Dizon DS, Strenger R, Legare RD, Theall KP, Graves TA, Gass JS, Kennedy TA Fenton MA (2009). Frequent pathologic complete responses in aggressive stages II to III breast cancers with every-4-week carboplatin and weekly paclitaxel with or without trastuzumab: a Brown University Oncology Group study. J Clin Oncol 27: 4693-4700.

8. Symmans WF, Peintinger F, Hatzis $C$, et al (2007). Measurement of residual breast cancer burden to predict survival after neoadjuvant chemotherapy. J Clin Oncol 25: 4414-4422.

9. von Minckwitz G, Huang C-S, Mano MS, et al (2019). Trastuzumab emtansine for residual invasive HER2-positive breast cancer. N Engl J Med 380:617-628.

10. van Ramshorst MS, van der Voort A, van Werkhoven ED, et al 2018). Neoadjuvant chemotherapy with or without anthracyclines in the presence of dual HER2 blockade for HER2-positive breast cancer (TRAIN-2): a multicentre, open-label, randomised, phase 3 trial. Lancet Oncol 19:1630-1640.

11. van der Voort A, van Ramshorst MS, van Werkhoven ED, et al (2020). Three-year follow-up of neoadjuvant chemotherapy with or without anthracyclines in the presence of dual HER2-blockade for HER2-positive breast cancer (TRAIN-2): A randomized phase III trial. J Clin Oncol 38, no.15 suppl. Abstract 501.

12. Rimawi MF, Cecchini RS, Rastogi P, et al (2017). A phase III trial evaluating pCR in patients with HR+, HER2-positive breast cancer treated with neoadjuvant docetaxel, carboplatin, trastuzumab, and pertuzumab (TCHP) +/- estrogen deprivation: NRG Oncology/NSABP B-52. Cancer Res 77: 4 suppl, Abstract S3-06.

13. von Minckwitz G, Schneeweiss A, Loibl S, et al (2014). Neoadjuvant carboplatin in patients with triple-negative and HER2-positive early breast cancer (GeparSixto; GBG 66): a randomised phase 2 trial. Lancet Oncol 15:747-756.

14. Untch M, Jackisch C, Schneeiweiss A, et al (2016). Nab-paclitaxel versus solvent-based paclitaxel neoadjuvant chemotherapy for early breast cancer (GeparSepto - GBG 69): a randomised phase 3 trial. Lancet Oncol 17: 345-356. 
15. Hurvitz SA, Martin M, Symmans WF, et al (2018). Neoadjuvant trastuzumab, pertuzumab, and chemotherapy versus trastuzumab emtansine plus pertuzumab in patients with HER2-positivw breast cancer (KRISTINE): a randomized, open-label, multicenter, phase 3 trial. Lancet Oncol 19:115126.

\section{Tables}

\section{Table 1}

Treatment Delivery ( $\mathrm{n}=30$ patients)

\begin{tabular}{|ccccccccccc|}
\hline Drug & \multicolumn{1}{c|}{ Number of patients receiving that number of doses } \\
\hline \# Doses Delivered & 18 & 17 & 16 & 15 & 13 & 12 & 10 & & \\
\hline Paclitaxel & 15 & 6 & 1 & 2 & 1 & $4^{*}$ & $1^{*}$ & & \\
\hline \# Doses Delivered & 18 & 17 & 15 & 13 & 12 & 10 & 8 & 7 & 6 \\
\hline Carboplatin & 10 & 8 & 2 & 1 & $3^{*}$ & 2 & $2^{*}$ & $1^{*}$ & 1 \\
\hline \# Doses Delivered & 6 & 5 & 4 & & & & & & \\
\hline Trastuzumab & 22 & 3 & $5^{*}$ & & & & & & \\
\hline \# Doses Delivered & 6 & 5 & 4 & 3 & 2 & 1 & & & \\
\hline Pertuzumab & 18 & 3 & $5^{*}$ & 1 & 2 & 1 & & & \\
\hline
\end{tabular}

*includes 2 patients who discontinued treatment and went to surgery and 3 patients switched to AC after week 12 (two for having achieved less than a cPR at re-staging and one for toxicity)

Paclitaxel dose reductions: 8 for neuropathy, 1 for neutropenia, 1 for anemia

Carboplatin discontinuation: 2 for HSR, 1 for diarrhea, 1 for neutropenia

Pertuzumab discontinuation: 6 for diarrhea 
Table 2

Toxicities - N (\%)

\begin{tabular}{|lllll|}
\hline Toxicity & Grade 1 & 2 & 3 & 4 \\
\hline ANC & NR & $10(33 \%)$ & $16(53 \%)$ & $1(3 \%)$ \\
\hline Thrombocytopenia & NR & $5(17 \%)$ & $1(3 \%)$ & 0 \\
\hline Anemia & NR & $22(73 \%)$ & $4(13 \%)$ & 0 \\
\hline Diarrhea & $11(37 \%)$ & $13(43 \%)$ & $4(13 \%)$ & $1(3 \%)$ \\
\hline Vomiting & NR & $1(3 \%)$ & 0 & 0 \\
\hline Neuropathy & NR & $5(17 \%)$ & 0 & 0 \\
\hline
\end{tabular}

ANC $=$ Absolute Neutrophil Count; NR $=$ Not recorded

Table 3

Pathologic Response Rates

\begin{tabular}{|llll|}
\hline Patient group & $\mathrm{N}$ & $\mathrm{pCR}-\mathrm{N}(\%)$ & $(95 \% \mathrm{Cl})$ \\
\hline All & 30 & $23(77 \%)$ & $(58-90 \%)$ \\
\hline Patient who received wPCbTP only & 27 & $21(78 \%)$ & \\
\hline ER-negative & 14 & $12(86 \%)$ & $(57-98 \%)$ \\
\hline ER-positive & 16 & $11(69 \%)$ & $(41-89 \%)$ \\
\hline Operable (Stage IIA-IIIA) & 22 & $19(86 \%)$ & $(65-97 \%)$ \\
\hline Inoperable (Stage IIIB-C) & 8 & $4(50 \%)$ & $(16-84 \%)$ \\
\hline Clinical CR after week 12 & 11 & $9(82 \%)$ & RCBI-1, RCBII-1 \\
\hline Clinical PR after week 12 & 14 & $12(86 \%)$ & RCBI-1, RCBII-1 \\
\hline Clinical MR/SD after week 12 & 5 & $2(40 \%)$ & RCBI-1, RCBII-2 \\
\hline pCR + RCBI & 30 & $26(87 \%)$ & $(69-96 \%)$ \\
\hline
\end{tabular}


Table 4

Comparison with other Neoadjuvant Trials with carboplatin and dual HER2-targeted therapy

\begin{tabular}{|c|c|c|c|c|c|}
\hline Study & Regimen & $\mathbf{N}$ & $\begin{array}{l}\text { pCR } \\
\text { (All) }\end{array}$ & $\begin{array}{l}\text { pCR } \\
\text { (ER-) }\end{array}$ & $\begin{array}{l}\text { pCR } \\
\text { (ER+) }\end{array}$ \\
\hline BrUOG 308 & $\begin{array}{l}\text { wPwCbTP x 18wks (or x } 12 \text { wks } \rightarrow \\
\text { ACx4) }\end{array}$ & 30 & $77 \%$ & $86 \%$ & $69 \%$ \\
\hline \multirow[t]{2}{*}{ TRAIN-2 $(10,11)$} & FECTP $3 \rightarrow w P q 3 C b T P \times 6$ cycles & 212 & $67 \%$ & $89 \%$ & $51 \%$ \\
\hline & wPq3CbTP $\times 9$ cycles & 206 & $68 \%$ & $84 \%$ & $55 \%$ \\
\hline $\begin{array}{l}\text { TRYPHAENA } \\
(4,5)\end{array}$ & Txq3CbTP x 6 & 76 & $52 \%$ & NR & NR \\
\hline \multirow{2}{*}{$\begin{array}{l}\text { NSABP B-52 } \\
(12)\end{array}$} & Txq3CbTP x 6 & \multirow[t]{2}{*}{308} & NA & NA & $41 \%$ \\
\hline & Txq3CbTP x 6 + Estrogen Deprivation & & NA & NA & $46 \%$ \\
\hline \multirow[t]{2}{*}{ GeparSixto (13) } & wPwnPLDTL x 18wks & 108 & $37 \%$ & NR & NR \\
\hline & wPwnPLDTL+wCb x 18wks & 129 & $33 \%$ & NR & NR \\
\hline \multirow[t]{2}{*}{ GeparSepto (14) } & wPTP $x 12$ wks $\rightarrow$ ECTP & 197 & $54 \%$ & $67 \%$ & $50 \%$ \\
\hline & wnPTP $x$ 12wks $\rightarrow$ ECTP & 199 & $62 \%$ & $78 \%$ & $56 \%$ \\
\hline Kristine (15) & Txq3CbTP x 6 (control arm) & 223 & $56 \%$ & $73 \%$ & $44 \%$ \\
\hline
\end{tabular}

$\mathrm{wP}=$ weekly paclitaxel; $\mathrm{wCb} / \mathrm{q} 3 \mathrm{Cb}=$ weekly/q3week carboplatin; $\mathrm{Tx}=$ docetaxel; $\mathrm{wnP}=$ weekly nabpaclitaxel; $\mathrm{T}=$ trastuzumab; $\mathrm{P}=$ pertuzumab; $\mathrm{L}=$ laptinib; $\mathrm{AC}=$ doxorubicin/cyclophosphamide; $\mathrm{FEC}=$ fluorouracil/epirubicin/cyclophosphamide; $E C$ = epirubicin/cyclophosphamide wnPLD = weekly nonpegylated liposomal doxorubicin

\section{Figures}




\section{BrUOG 308 - Schema}

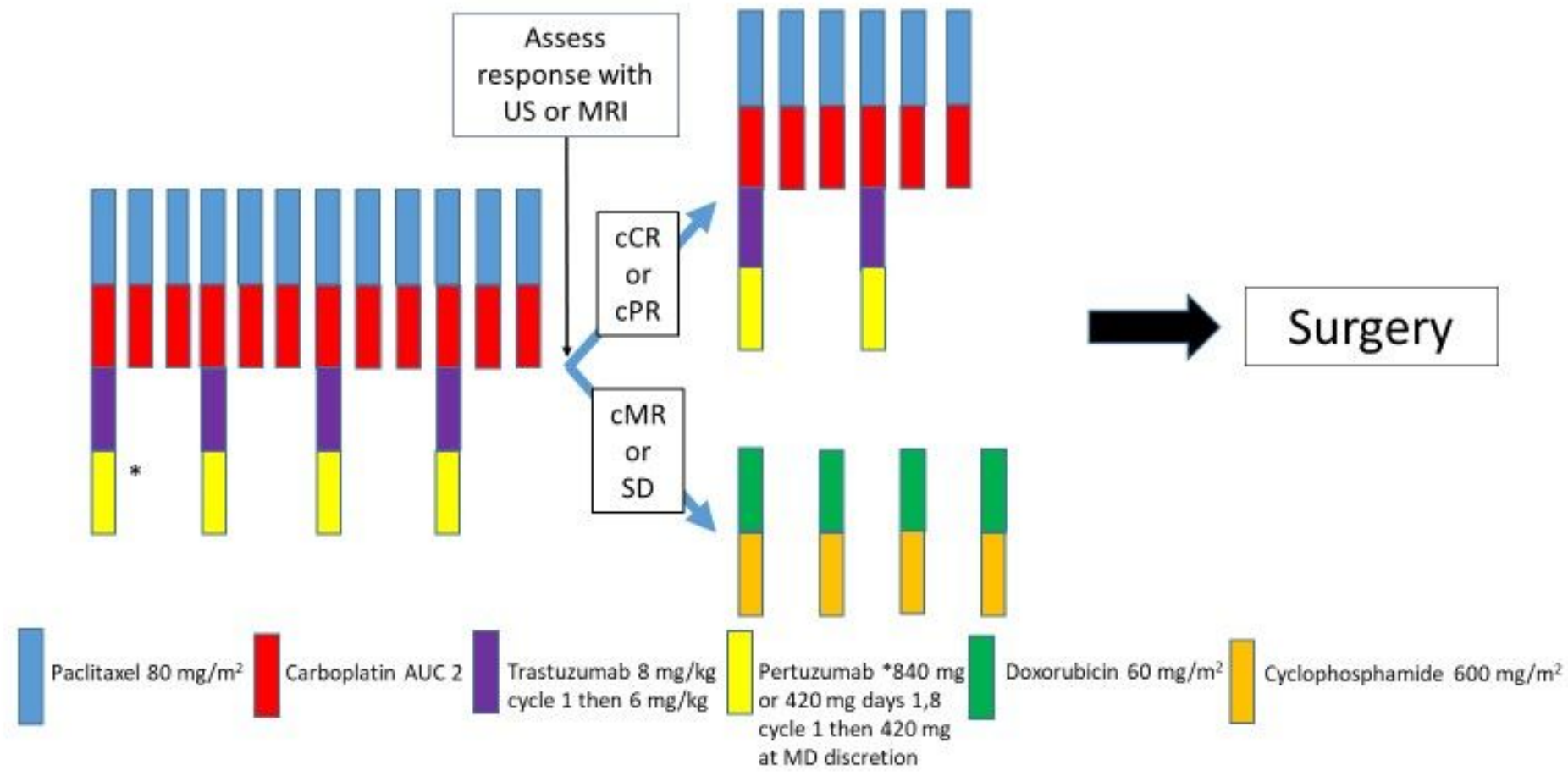

\section{Figure 1}

Study Schema. BrUOG 308 schema - US = Ultrasound; MRI = Magnetic Resonance Imaging (of the breasts) $; \mathrm{cCR}=$ Clinical Complete Response; $\mathrm{CPR}=$ Clinical Partial Response; $\mathrm{cMR}=$ Clinical Minimal Response; $\mathrm{SD}=$ Stable Disease 


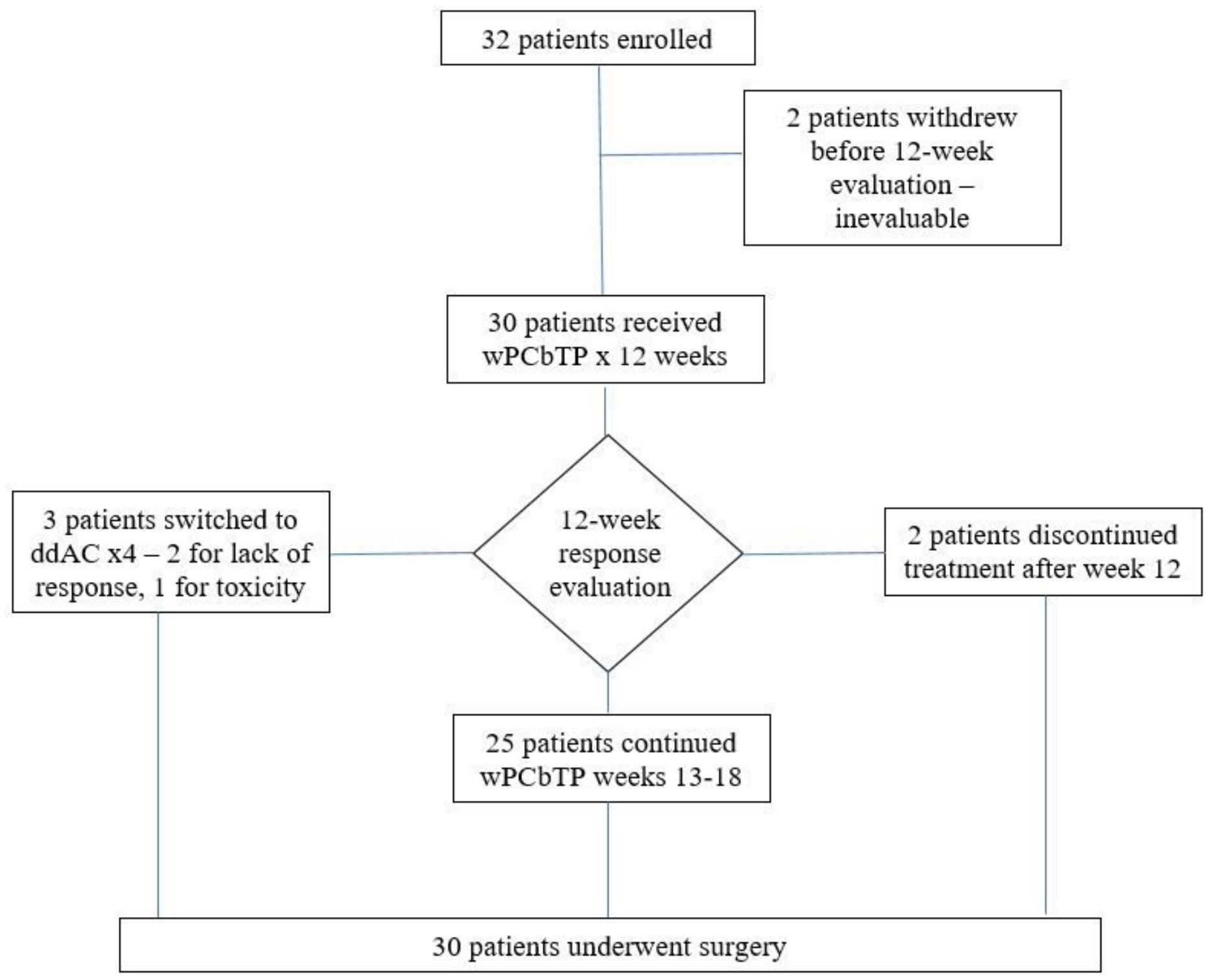

Figure 2

CONSORT Diagram. BrUOG 308 CONSORT Diagram 